\title{
LARGE-SCALE EJECTION STRUCTURES IN THE GALACTIC CENTER
}

\author{
Yoshiaki SOFUE \\ Institute of Astronomy \\ The University of Tokyo \\ Mitaka, Tokyo 181, Japan
}

\begin{abstract}
Radio continuum observations of the galactic center region have revealed a number of vertical structures running across the galactic plane, most of which are reasonably attributed either to vertical magnetic fields or to energy release out of the galactic plane. We review the observed radio structures and discuss their properties and origins with a particular attention to the unusual manifestation of energy release in the galactic center. The relation of the continuum structures to the expanding and/or contracting molecular gas rings is also discussed.

Keywords: Activity; Galactic center; Jets; Magnetic field;
\end{abstract}

\section{INTRODUCTION}

Recent progress in the study of the galactic center region owes its significant part to radio and infrared observations mainly due to the negligible or small absorption in spite of the long path through the dense interstellar medium near the galactic plane. Optical and UV lights are significantly absorbed by the interstellar gas and dust. Soft $\mathrm{X}$ rays are also absorbed by the large line-of-sight column density of hydrogen, $N_{\mathrm{H}} \sim 10^{23} \mathrm{~cm}^{-2}$. Hard $\mathrm{X}$ rays ( $>$ a few $\mathrm{keV}$ ) are another tool to observe the galactic center, while limited observations have been done so far (e.g. Watson et al 1981). Gamma rays can be used to investigate high energy phenomena in the nucleus, but the present-day facilities are not sufficient in resolution to study the structural details.

In this paper we review radio continuum observations of the galactic center with a particular regard to large-scale (larger than a few tens of parsecs) extended features. The radio features are often perpendicular to the galactic plane and are a mixture of various components with different emission mechanisms (Fig. 1, 2). We first discuss the properties of the radio emission, reviewing some method to distinguish thermal and nonthermal emissions. We then summarize various exotic structures with peculiar morphology, which are mostly perpendicular to the galactic plane, and discuss them in relation to vertical magnetic fields and to manifestation of energy release out of the nuclear disk. We discuss the relation of these enigmatic radio structures to various activities associated with ejection of matter from the center region. The expanding and/or contracting rings of molecular gas as observed 


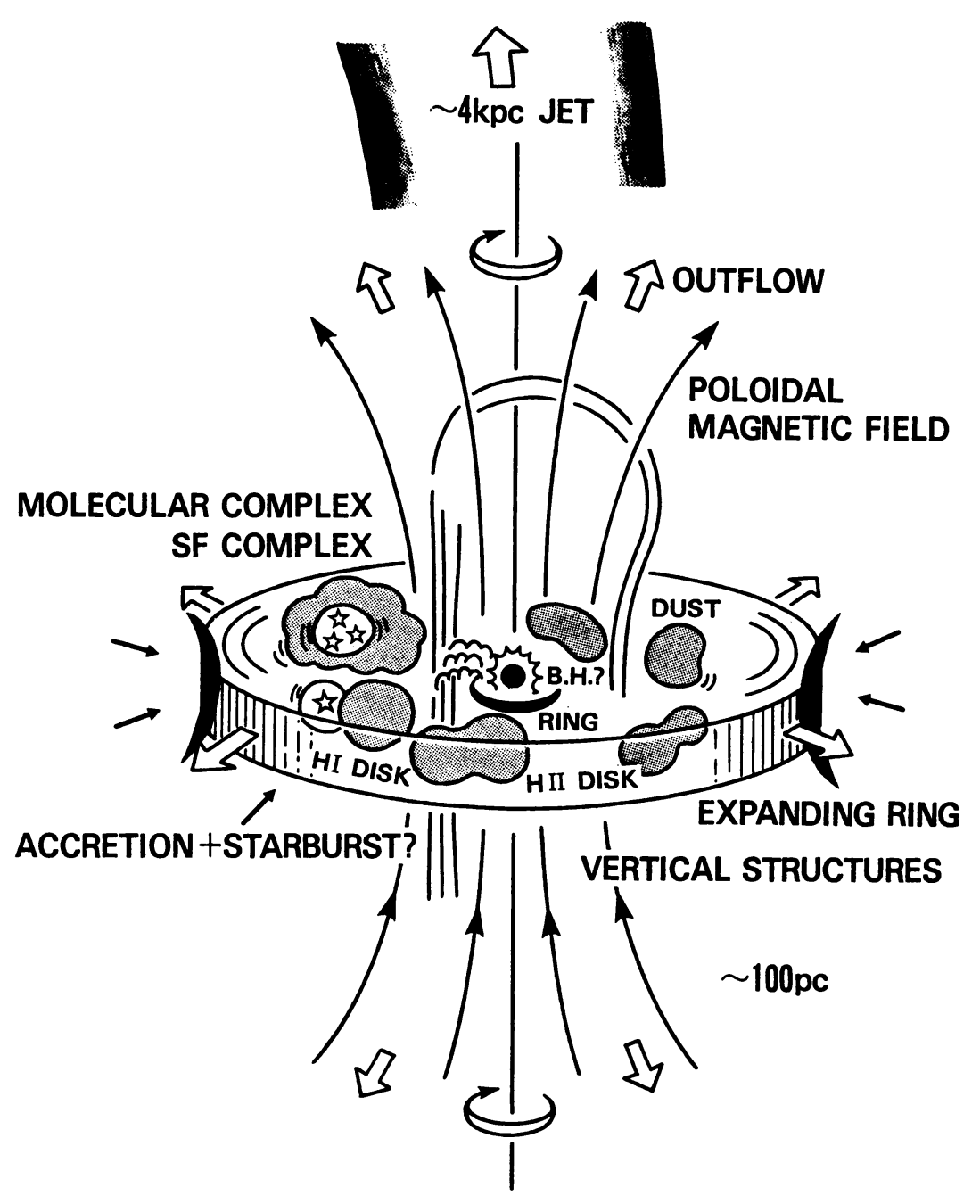

GALACTIC CENTER?

Fig. 1: Schematic view of "the galactic center" which is a mixuture of various emission components. Many structures are found extending perpendicular to the disk plane. 

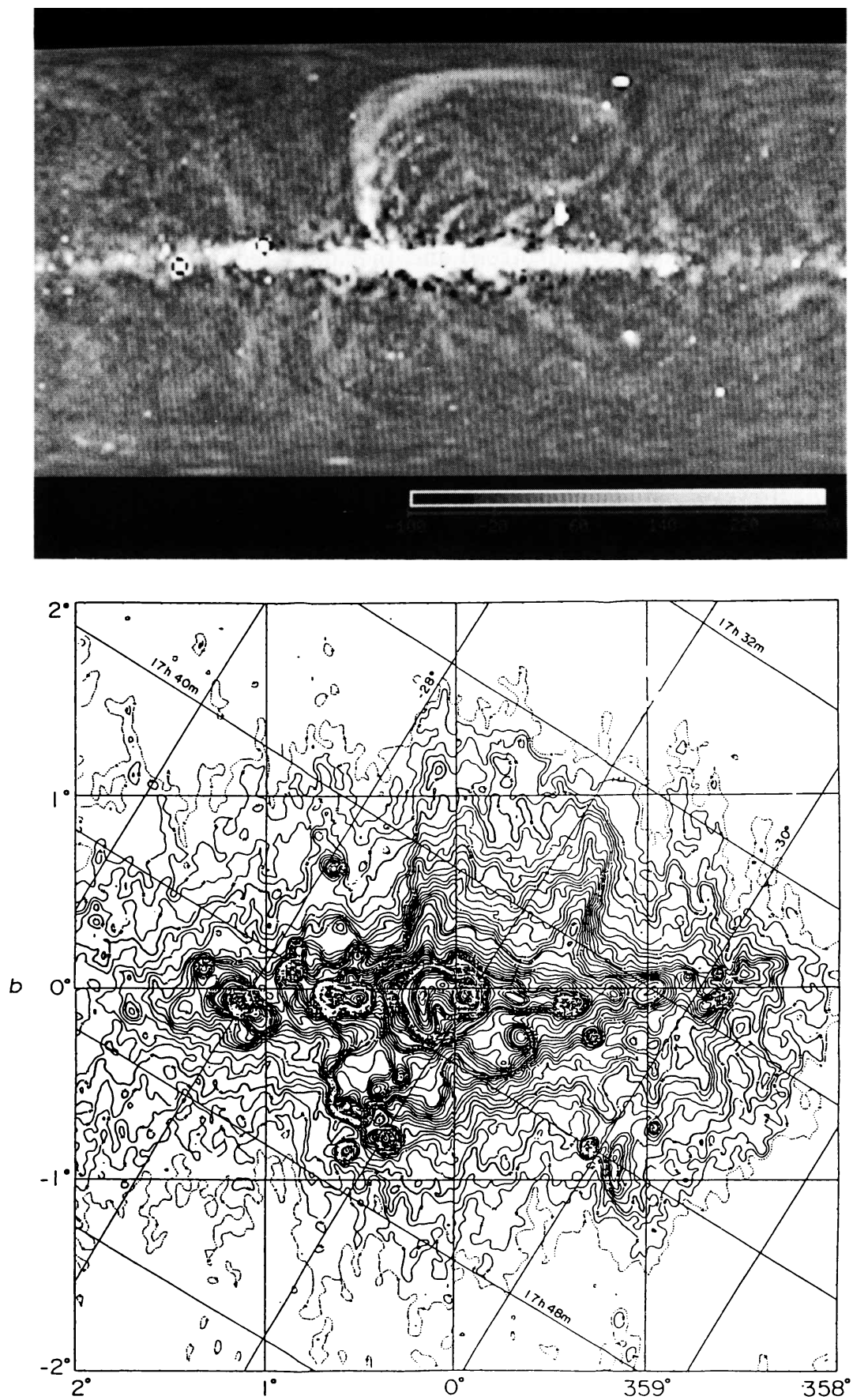

Fig. 2: Radio continuum Milky Way after subtraction of diffuse components from the all-sky $408 \mathrm{MHz}$ survey of Haslam et al (1982) (top), and a $10 \mathrm{GHz}$ map of the central $4^{\circ}$ from the Nobeyama survey (bottom). Various features are seen not lying in the galactic plane, but vertical to the galactic plane. 
in the molecular lines in the $\mathrm{mm}$ and sub-mm wave ranges will be discussed in relation to these ejection structures.

The readers may refer to several review articles in this issue as well as those by Oort (1977, 1986); Lo (1986); Brown and Listzt (1984); and Genzel and Townes (1987).

\section{PROPERTIES OF RADIO CONTINUUM EMISSION}

The radio continuum emission from the galactic center region of $\sim 3^{\circ} \times 3^{\circ}$ is a mixture of nonthermal (synchrotron) and thermal (free-free) emissions. In order to investigate the various observed structures it is essential to clarify their emission mechanisms. We here summarize some methods to separate these two emission components.

\subsection{Spectral indices}

The most conventional way to investigate the emission mechanism of radio continuum emission is to study the spectral index. Thermal emission shows a flat spectrum of $\alpha=-0.1$ with $S \propto \nu^{\alpha}$, while nonthermal emission usually shows steep spectrum. However, studying spectral indices derived from radio data in large frequency separation, from $845 \mathrm{MHz}$ to $43 \mathrm{GHz}$, Reich et al (1987b) have shown that the spectral index in the radio Arc and bridge region near Sgr A is mostly flat or even inverted (positive) (Fig. 3). Sofue (1985) has also shown that the spectral index in the central $3^{\circ}$ region is flat. The flat spectra are obtained in nearly all regions where strong linear polarization has been detected (see below for polarization).

The flat spectrum obtained near the galactic center must no longer be taken, therefore, as an indicator of thermal emission. We note that many active galactic nuclei in extragalactic systems often show flat radio spectra for their nonthermal characteristics. However, the question why the spectra are so flat, or how highenergy electrons are supplied so efficiently in such a wide area of the galactic center, is not answered as yet.

We may only suggest that the galactic center region might be a huge Crab-like object: As is well known, a Crab-like SNR is a nebulosity associated with a central engine, such as a pulsar or a neutron star supplying high-energy electrons to create the flat-spectrum radio emission (e.g. Weiler 1983). The galactic center might be in a similar situation in the sense that fresh cosmic rays responsible for the flat radio spectra are supplied from some central object and are trapped in strong magnetic fields.

\subsection{Linear Polarization}

A direct and more convincing way to distinguish synchrotron radiation is to measure the linear polarization. However, an extremely high Faraday rotation caused by the dense interstellar matter and long path length through the galactic plane often depolarizes the emission by the finite-beam and finite-bandwidth effects, which makes the measurement difficult. This difficulty has been resolved by the development of a multi-frequency, narrow-band Faraday polarimeter (Inoue et al 1984) as well as by high-resolution and high-frequency observations using the VLA (Yusef-Zadeh et al 1986; Inoue et al 1988). Very large rotation measure $(R M>\sim$ $\left.10^{3} \mathrm{rad} \mathrm{m}^{-2}\right)$ and high degree $(10-30 \%)$ of polarization have been observed along the radio Arc and on the eastern ridge of the Galactic Center Lobe (Inoue et al 1984; Tsuboi et al 1986; Seiradakis et al 1985; Sofue et al 1986). 

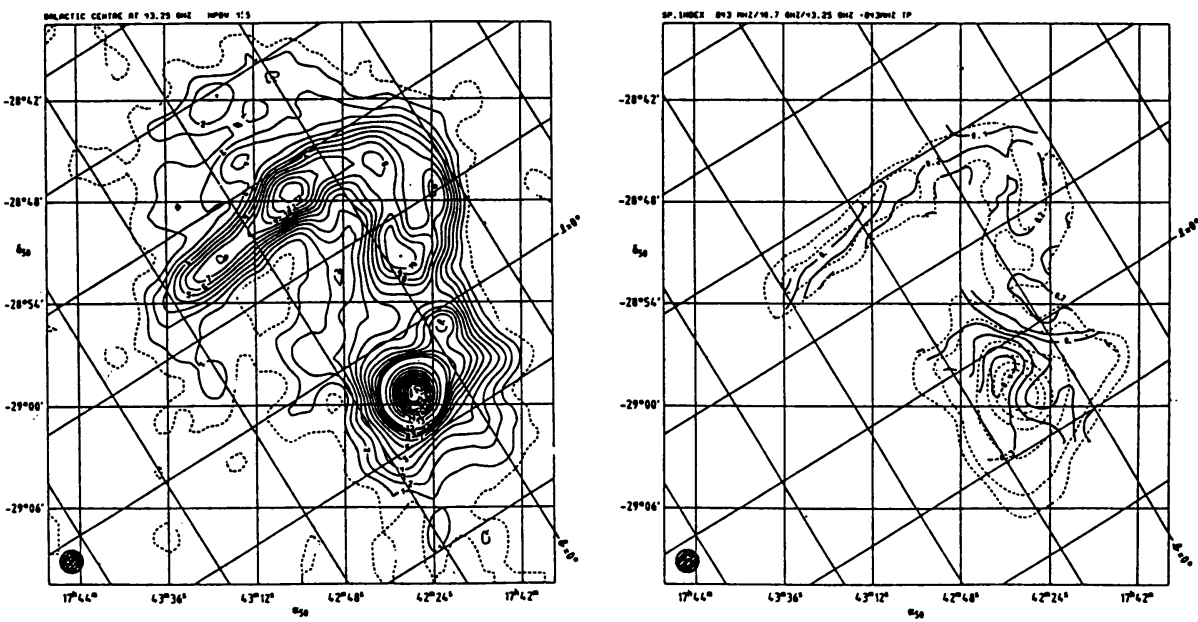

Fig. 3: Spectral index distribution in the radio arc and near Sgr A as detemined from data at $843 \mathrm{MHz}, 10 \mathrm{GHz}$ and $43 \mathrm{GHz}$ (Reich et al 1987). Note that the index is flat or even inverted despite the nonthermal emission characteristics.
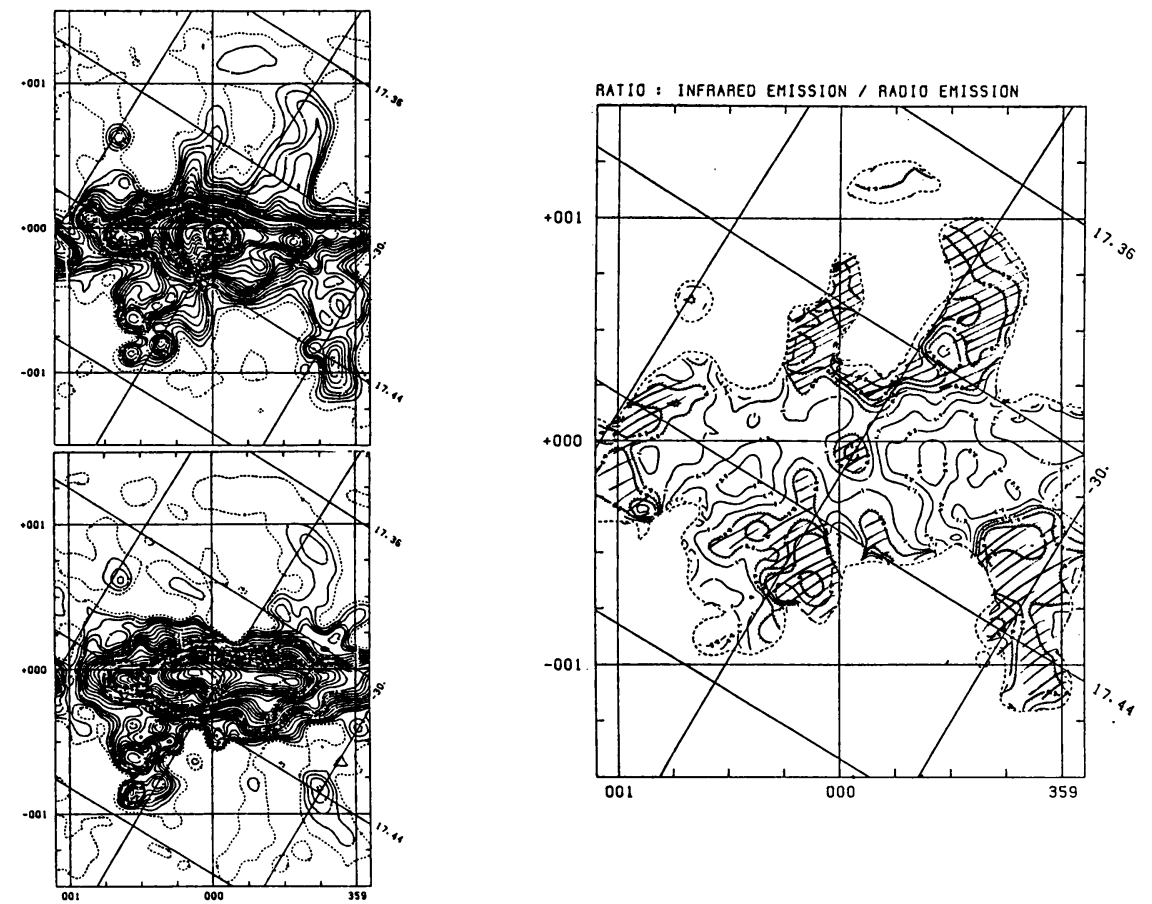

Fig. 4: $11-\mathrm{cm}$ radio emission (left top), $60 \mu \mathrm{mFIR}$ emission (left bottom), and the IR-to-radio ratio, $R=I_{60 \mu \mathrm{m}} / I_{11 \mathrm{~cm}}$ (right), for the central $2^{\circ} \times 3^{\circ}$ region. Hatched regions have small $R$ values and are nonthermal, while non-hatched regions have large $R$ values $\left(\sim 10^{3}\right)$ and are mostly thermal. 
At high frequencies the Faraday depolarization becomes less effective. Reich (1988) has reported the detection of very high of polarization, $p \sim 50 \%$, along the Arc at $32 \mathrm{GHz}$. This high polarization is nearly equal to the maximum polarization degree, $p_{\max }=(\alpha+1) /(\alpha+7 / 3) \simeq 47 \%$ for $\alpha \simeq+0.2$ of the Arc. This fact implies that the magnetic field is almost perfectly ordered. This is also consistent with the VLA observations showing straight filaments suggestive of highly ordered magnetic field (Yusef-Zadeh et al 1984).

From these observations of linear polarization it is clear that the radio emission near the galactic center, in particular around the radio Arc and on the GCL, is nonthermal despite their flat or inverted spectra.

\subsection{Infrared-to-Radio Ratio}

The separation of thermal and nonthermal radio emission can be done in a more efficient way even for the regions where no polarization data are available. The method uses comparison of the far IR (e.g. $60 \mu \mathrm{m}$ data from IRAS survey) and radio surveys : thermal emission regions, mostly composed of HII gas, have high IR-to-radio ratio, $R=I_{\mathrm{FIR}} / I_{\mathrm{R}} \simeq 10^{3}$. This is because the thermal gas is mixed with dust grains heated by interstellar UV light from OB stars (Fürst et al 1987; Reich et al 1987a). On the other hand, nonthermal emission regions, such as supernova remnants, have small IR-to-radio ratio, $R=0 \sim 300$.

Using these characteristics we have been able to distinguish thermal and nonthermal emission regions in a wide area near the galactic center (Reich et al 1987). Fig. 4 shows the radio continuum and FIR $(60 \mu \mathrm{m})$ images, and the distribution of the IR-to-radio ratio $R$ between $60 \mu \mathrm{m}$ and $2.7 \mathrm{GHz}$ for the central $2^{\circ} \times 3^{\circ}$. The hatched areas have small $R$ and are nonthermal regions, while non-hatched areas are thermal, mostly HII regions.

The region near the galactic plane is dominated by thermal emission and many strong radio sources like Sgr B2 appear thermal (HII) (see, e.g., Mezger and Pauls 1979). These regions are closely associated with dense molecular clouds (Bally et al 1987) and therefore related to star formation from the clouds.

On the other hand we find many of the prominent features like the Radio Arc, Sgr A and regions high above the galactic plane including the GCL(galactic center lobe) are nonthermal. Note that the western ridge of the GCL is shown to be clearly nonthermal. We note that the western ridge has been recently resolved into thin filaments (Yusef-Zadeh 1988), which suggests that the ridge is composed of magnetic field. Some supernova remnants (G359.0-0.5, G359.1-0.9, G0.6+0.05) are also seen in Fig. 4 as small $R$-value regions.

\section{OUT-OF-PLANE RADIO STRUCTURES}

The galactic center region has been mapped at various frequencies [e.g. $80 \mathrm{MHz}$ (LaRosa and Kassim 1985); $160 \mathrm{MHz}$ (Dulk and Slee 1974; Yusef-Zadeh et al); 327 $\mathrm{MHz}$ (Yusef-Zadeh et al ); $408 \mathrm{MHz}$ (Little 1974; Haslam et al 1982); $610 \mathrm{MHz}$ (Downes et al 1978); $843 \mathrm{MHz}$ (Mills and Drinkwater 1984); $1.5 \mathrm{GHz}$ (Yusef-Zadeh et al 1984; Liszt 1986); $2.7 \mathrm{GHz}$ (Reich et al 1984); $5 \mathrm{GHz}$ (Altenhoff et al 1979); 10 GHz (Pauls et al 1976; Handa et al 1987); $15.5 \mathrm{GHz}$ (Kapitzky and Dent 1974); 32 $\mathrm{GHz}$ (Reich 1988); $43 \mathrm{GHz}$ (Sofue et al 1986)]. The radio continuum mappings have revealed various exotic features of peculiar morphology. Many of them appear to lie not parallel to the galactic plane, but run roughly perpendicular to the disk. They 
are suspected to be the manifestation of energy release at the center. Some of them are most likely related to the magnetic field. We here summarize the prominent radio continuum structures of scales larger than a few tens of parsecs. For smaller structures the readers may refer to other review articles in this issue (Becklin 1988; Ekers 1988; Genzel 1988; Goss 1988; Güsten 1988; Lacy 1988; Lo 1988; Morris 1988; Rieke 1988; Yusef-Zadeh 1988).

\subsection{Bridge and Arched Filaments (10-30 pc)}

Complex filaments are extending from Sgr A toward the north, composing a bridge between the radio Arc and Sgr A (Yusef-Zadeh et al 1984; Yusef-Zadeh and Morris 1988). Detection of hydrogen recombination lines (Pauls et al 1976; Yusef-Zadeh et al 1986) and the association of molecular gas at negative velocities (Serabyn and Güsten 1986; 1987) indicate its thermal characteristics, and the structures are called the thermal arched filaments. However, the physical connection of the arc and the bridge is still controversial (Yusef-Zadeh and Morris 1987).

Detection of large Faraday rotation toward the bridge region suggests the existence of a magnetic field along the filaments (Sofue et al 1987). A magnetoionic jet model in which the gas is flowing out of Sgr A and collides with the ambient poloidal magnetic field at the radio Arc has been proposed (Sofue and Fujimoto 1987; Heyvaerts et al 1988). However, Serabyn and Güsten (1987) argue that the gas is flowing toward the center and an accretion model has been proposed by analysing the velocity field.

The complex structure in the bridge ( thermal, arched filaments) indicate high turbulent motion inside the bridge. In fact the velocity dispersion, as high as 30-50 $\mathrm{km} \mathrm{s}^{-1}$, is much higher than the systemic motion of the bridge, $-20 \sim-30 \mathrm{~km} \mathrm{~s}^{-1}$ . The increase of the velocity dispersion (60-70 $\left.\mathrm{km} \mathrm{s}^{-1}\right)$ near the radio Arc suggests a dynamical interaction of the bridge with the Arc. Yusef-Zadeh and Morris (1988), based on their high-resolution maps, argue that the Arc (straight filaments) and the arched filaments are interacting with each other.

\subsection{Radio Arc ( $30 p c)$}

This remarkable feature was originally found in the radio continuum survey maps of the galactic plane (Downes et al 1978), and has been resolved into many straight filaments with the use of VLA (Morris and Yusef-Zadeh 1987, 1988; YusefZadeh et al 1984; Yusef-Zadeh 1986; 1988). The straight filaments run perpendicular to the galactic plane, and extend more than $\sim 100 \mathrm{pc}$ toward positive latitudes. The mid point of the arc is strongly polarized $(\sim 20 \%)$ in the microwave range, and this polarized spot shows a high Faraday rotation $\left(R M \sim\right.$ a few $\left.10^{3} \mathrm{rad} \mathrm{m}^{-2}\right)$ (Inoue et al 1984; Tsuboi et al 1986; Sofue et al 1987; Yusef-Zadeh and Morris 1988).

The detection of theoretically maximum polarization of $50 \%$ along the Arc (Reich 1988) indicates a highly aligned magnetic field and is consistent with the straight filamentary structure. The transverse magnetic field direction, as detemined from the intrinsic polarization angles, is parallel to the arc ridge, namely the field is vertical to the galactic plane. The upper and lower sides of the polarized spot are strongly depolarized, due to an extremely high internal Faraday rotation (possibly $R M>10^{4} \mathrm{rad} \mathrm{m}^{-2}$ ).

3.3 Polarized plumes (50-100 pc) 
The higher latitude extensions of the Arc, both toward positive and negative latitudes for more than $100 \mathrm{pc}$, are also polarized and are called polarized plumes (Tsuboi et al 1986; Yusef-Zadeh and Morris 1988). The highest degree of polarization reaches as high as $20 \%$. The magnetic field directions are parallel to the radio arc, or vertical to the galactic plane. High Faraday rotation (1000-2000 $\mathrm{rad} \mathrm{m}^{-2}$ ) has been detected (Tsuboi et al 1986; Sofue et al 1987). The sense of rotation measure reverses from the positive to negative latitude sides, indicating a reversal of the line sight component of the magnetic field above and below the galactic plane. The field strength is estimated to be 10-100 $\mu$ G. Sofue et al (1986) suggest that the polarized spot and the plumes are parts of a large-scale poloidal magnetic field twisted by the disk rotation (see next section).

\subsection{Sgr C filament ( $10 \mathrm{pc})$}

The third brightest source near the galactic center, Sgr C, located at $l=-0.6^{\circ}$, has been resolved into thin filament of 15 pc length (Liszt 1985). The filament is as thin as a few arc second and is comparable to the straight filaments in the Arc and the threads. The filament is again almost perpendicular to the galactic plane, suggestive of a vertical magnetic field running across the galactic disk. Its positive latitude extension merges the the western ridge of the galactic center lobe (see below), while the southern extension is weak.

\subsection{Threads (10-30 pc)}

High-sensitivity VLA maps reveal thin ( $\sim 0.5 \mathrm{pc}$ wide) and straight filaments, called the threads. They run across the Sgr A region and the galactic plane (Morris and Yusef-Zadeh 1985; Yusef-Zadeh 1988). They are roughly perpendicular to the disk plane, and seem to be not closely connected to $\mathrm{Sgr} \mathrm{A}$, the Bridge (Arched filaments), or to the Arc. The threads intersect the other features without any clear interaction. From the very thin and straight appearence the threads are likely magnetic structures, and the vertical nature is consitent with a large-scale poloidal field in the central region (see below).

\subsection{Low-energy jet and a bubble (30-40 pc)}

At low frequencies, $160 \mathrm{MHz}$, a jet-like feature is found to emanate from Sgr A-East toward negative latitude for 30-40 pc (Yusef-Zadeh et al 1986). The feature is roughly perpendicular to the disk plane and may be a jet from the nucleus. On the southern extension of this jet a shell-like object of diameter $\sim 40 \mathrm{pc}$ is found. This peculiar feature may be a magnetized bubble of cosmic rays produced by the ejection (injection into interstellar space) of the jet (Sofue 1988).

\subsection{Galactic center lobe (100-200 pc)}

In larger scales, $100-200 \mathrm{pc}$, we find the galactic center lobe (GCL), which is composed of a giant lobe structure extending vertically over the galactic plane (Sofue and Handa 1984; Sofue 1985). The eastern ridge of the lobe is an extension from the radio Arc and is strongly polarized, and observed as the northern polarized plumes. The magnetic field is shown to run parallel to the ridge. The ridge is also extends toward negative latitude, where a symmetric polarized plume is found. The western ridge emerges from Sgr C and is clearly the extension of the VLA filament. Yusef-Zadeh (1988) reports the detection of filament and polarization in the western ridge, indicating the existence of magnetic field.

An MHD acceleration model in which the gas is accelerated via a twist of poloidal magnetic field by the accreting gas disk has been proposed for the forma- 
tion of the lobe structure (Shibata 1988). Heyvaerts et al (1987) have proposed a magnetic loop model where the lobe structure is produced by magnetized ejecta from the central massive object. An alternative model is that the lobe is due to an expanding shock caused by an explosive event in the nucleus (Sofue 1984; Umemura et al 1988).

\subsection{Galactic center jet $(\sim 4 k p c)$}

By applying the background filtering technique to the $408-\mathrm{MHz}$ all-sky survey data (Haslam et al 1982), we have shown that there exist numerous spurs of length $10-30^{\circ}$ emanating from the galactic disk in the central $\sim 60^{\circ}$ (Fig.2: Sofue 1988). The spurs are roughly perpendicular the galactic plane, and are crowded more in the central $\sim 30^{\circ}$ than in the outer regions. This suggests that the vertical spurs might be associated with some activity in the central region. These structures might be ridges composed of vertically blown-up matter from the galactic disk, likely associated with the magnetic field.

Among the spurs, the one emanating from the galactic center toward positive latitude, $b \sim 25^{\circ}$, has been noticed by Haslam et al (1981) and by Sofue et al (1988). Sofue et al (1988), with the detection of the ridge connecting the spur to the galactic center by their $1408 \mathrm{MHz}$ observations, interpret this as a jet from the galactic center: This feature, which is 4-kpc long and some 200-pc in diameter, may be cylindrical in shape and extends roughly perpendicular to the galactic plane. This structure might be a jet (or the remnant of a relativistic beam from the nucleus), or it might be magnetic tornado produced by the differential rotation between the halo and the nuclear disk.

\subsection{HI Chimney (a few kpc)}

Detection of a long jet-like feature observed in the HI emission has been reported (Kundt 1987). The feature, called an "HI chimney", appears in specific velocity ranges (at $v \sim-60 \mathrm{~km} \mathrm{~s}^{-1}$ for $l>0, b>0$, while $v \sim 40 \mathrm{~km} \mathrm{~s}^{-1}$ for $l<0, b<0)$, extending from the central region both toward positive and negative latitudes in a symmetrical way for some tens of degrees (a few $\mathrm{kpc}$ ). The chimney is not positionally correlated with the Galactic Center Jet, and their relation to each other is open to question. We note that the galactic center jet seems not to be associated with any HI features. Kundt (1987) suggests that the HI chimney may be a remnant of ionized gas produced by a jet emanating from the galactic center as a result of some Seyfert-like activity.

\section{VERTICAL MAGNETIC FIELDS}

The radio continuum observations show various features extending roughly perpendicular to the galactic plane. The thin, straight morphology of the features suggests that the structures are deeply coupled with vertical magnetic fields.

\subsection{Poloidal fields}

The polarization observations of the galactic center region show that the field direction projected on the sky along the radio Arc and the eastern ridge of the galactic center lobe (polarized plumes), is perpendicular to the galactic plane (Yusef-Zadeh et al 1984; Inoue et al 1984; Tsuboi et al 1986; Sofue et al 1987).

Measurements of the Faraday rotation in the galactic center region show that the rotation measure (RM) reverses from the lower side of the galactic plane to the upper side, indicating the reversal of the line-of-sight component of the field. 

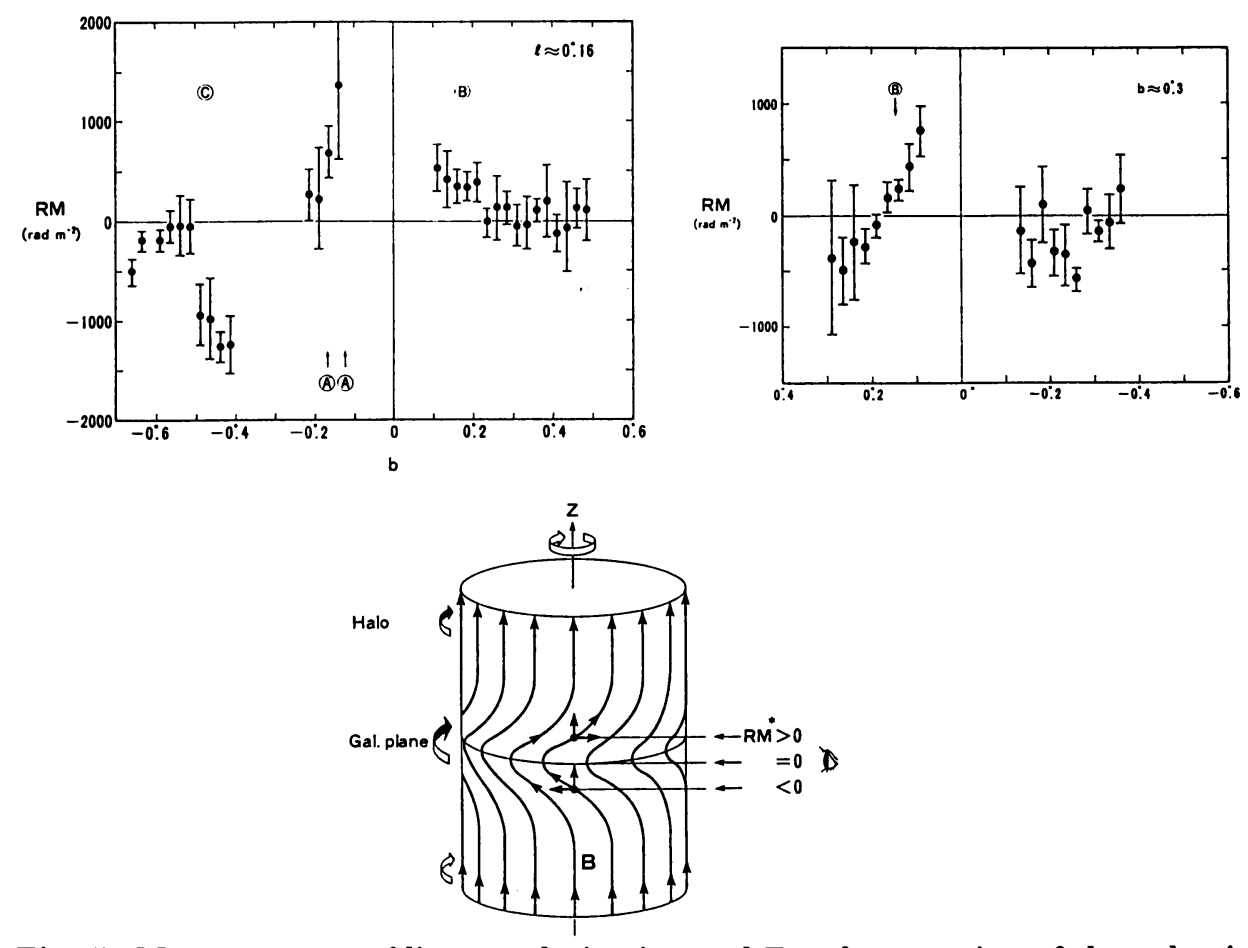

Fig. 5: Measurements of linear polarization and Faraday rotation of the galactic center region suggest a poloidal field twisted by the disk rotation.

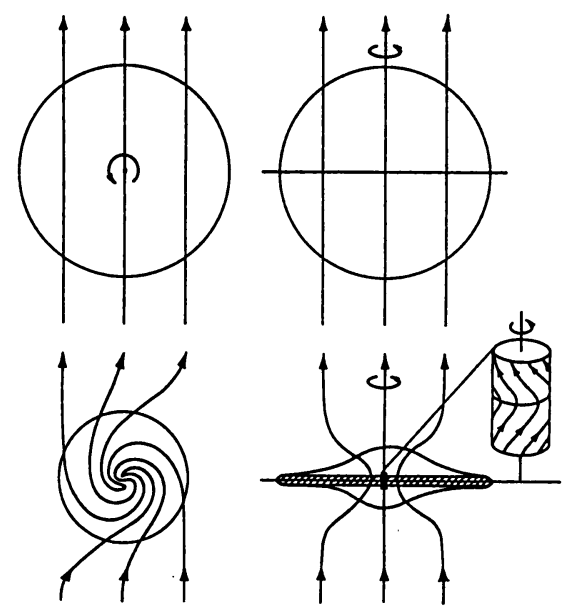

Fig. 6: Disk fields in spiral galaxies are dominated by bisymmetric open spiral configuration, which suggests a primordial origin of the disk field. Then the primordially vertical component must be accumulated somewhere near the galaxy center producing a strong poloidal field. 
The reversal of the RM is also observed across the rotation axis of galaxy ( $z$-axis) (Tsuboi et al 1986; Sofue et al 1987). From these facts a poloidal field model has been proposed in which the field lines are twisted by the disk rotation as illustrated in Fig. 5 .

On the other hand, the very straight nature of the filaments in the radio Arc might suggest that the field line is not bending but three-dimensionally straight. If this is the case, the reversal of RM may be attributed to a field reversal somewhere inbetween the Arc and the observer. The fact that the reversal is systematic, that the scale length of $\mathrm{RM}$ variation, $\sim 10^{\prime}$, is small, and that the $\mathrm{RM}$ amplitude is as large as $R M \sim 10^{4} \mathrm{rad} \mathrm{m}^{-2}$, suggests that the Faraday rotation is not likely occuring in the local interstellar space, but it may occur in the nuclear disk, which has a toroidal and/or radial magnetic field with its direction being systematically reversing from the lower to upper side of the plane.

\subsection{Magnetic fields in spiral galaxies}

Observations of linear polarization of nearby spiral galaxies show that the large scale configuration of the magnetic field in the disks of these galaxies is predominantly bisymmetric open-spiral (BSS): the field lines come into the galaxy from one edge of the disk, spiral around the center almost parallel to the spiral arms, and go out from the diametrically opposite edge of the disk (e.g. Sofue et al 1986).

The galactic dynamo theories (Parker 1972; Sawa and Fujimoto 1986; Fujimoto and Sawa 1987; Ruzmaikin et al 1985) have shown that such bisymmetric spiral pattern (mode 1) can be maintained by the dynamo action in the presence of turbulent diffusion. On the other hand, the theories equally show that magnetic field of circular and radial configuration (mode 0 ) can also be maintained in a steady state. According to the theories, even higher-order (mode $>1$ ) configurations can also be maintained and should equally exist in disk galalxies. Contrary to the prediction, observations show that the majority of the galaxies possess the BSS fields.

The fact that the BSS field dominates in spiral galaxies may be most simply explained if the field configuration is primordial in origin (Fig. 6) : An intergalactic primordial magnetic field was trapped when the protogalaxies formed, wound up by the differential rotation of the disk, and is maintained in a steady state through the dynamo mechanism in the presense of turbulent diffusion. The time scale of field annihilation and amplification through the galactic dynamo and turbulent diffusion in normal galaxy disks is of the order of $\tau_{\text {disk }} \sim d^{2} / l v \sim 10^{7 \sim 8} \mathrm{y}$, where $d \sim 100 \mathrm{pc}$ is the disk thickness, $l \sim 100 \mathrm{pc}$ is the eddy size of the turbulence and $v \sim$ a few tens of $\mathrm{km} \mathrm{s}^{-1}$ is the turbulent velocity. This time scale is comparable to or smaller than the time scale of differential rotation, so that a steady state is realized. Such a scenario of the evolution of the BSS field in the galaxy disk, however, takes into account only the component of primordial field parallel to the disk plane.

\subsection{Origin of vertical fields}

If we stand on the primordial origin hypothesis of galactic magnetic fields, we should inevitably consider the evolution of the vertical component of the primordial field. The time scale with which a magnetic field component perpendicular to the disk plane diffuses away from the galaxy may be estimated as $\tau_{\text {vert }} \sim r^{2} / l v \sim 10^{11} \mathrm{y}$, by taking $r \sim 10 \mathrm{kpc}$ with $r$ being the radius of the galaxy disk. This means that the vertical component trapped from the intergalactic space can never escape from the galaxy, but is transferred to the central region when the galaxy contraction proceeds. 
A rough estimation shows that the field strength of the vertical component in the galaxy is proportional to the surface mass density (Sofue and Fujimoto 1987), and a strong vertical field, some $10 \mu \mathrm{G}$, is expected to exist in the central region. On the other hand, the disk component parallel to the galactic plane annihilates in the central region more efficiently than in the outer region and a rather weaker disk field is expected there (Fujimoto and Tosa 1980).

This scenario of evolution of the galactic field predicts that a vertical field dominates in the central region, while the disk spiral field has the maximum away from the central region. This is consistent with the fact that our Galaxy possesses a twisted poloidal field in the center, while the disk field is strongest at $r \sim 5 \mathrm{kpc}$. Similar characteristics have been found in the spiral galaxy M31 (Berkhuijsen et al 1987): the central radio source shows polarization characteristics consistent with the vertical field: the projected field direction is perpendicular to the major axis, while the disk field, which is the superposition of ring and spiral, has the maximum at $r \sim 10 \mathrm{kpc}$.

\section{FORMATION OF THE VERTICAL STRUCTURES}

Some of the various scale vertical structures as observed in the radio continuum may be understood if the central few hundred pc has a strong poloidal magnetic field. Alternatively, some of them could be structures produced by some violent energy release at the center such as an explosion or a starburst. Here we briefly discuss possible formation mechanisms of the observed features. [For details, see Shibata (1988)].

\subsection{MHD Hypothesis}

The radio Arc, along which runs a straight vertical field, may be a part of the poloidal field illuminated by some ejection of matter and/or magnetized blobs from the nucleus (Sofue and Fujimoto 1987; Heyvaerts et al 1987; Heyvaerts 1988). The interaction of the arched filaments (bridge) and the Arc (Yusef-Zadeh and Morris 1988 ) suggests some dynamical interaction which may give rise to acceleration of cosmic rays. Cosmic rays accelerated there will then propagate along the magnetic tube toward high latitudes, where they emit highly polarized radio emission and are observed as the polarized plumes.

The field will be twisted by the accreting nuclear gas disk. The twist will then propagate toward the halo, accelerating the gas perpendicular to the disk plane. This will result in a cylindrical outflow of gas and cosmic rays, which will account for the formation of the galactic center lobe (Uchida and Shibata 1986; Shibata and Uchida 1987; Shibata 1988) (Fig. 7). The Sgr C filament is a part of the western ridge of the GCL, where a vertical field yields the thin, filamentary structure.

The large-scale poloidal field running through the galactic halo will also be twisted by the differential rotation beween the disk and halo gases. The twist will amplify the field strength near the rotation axis, and will be observed as the galactic center jet of 4-kpc length (Sofue et al 1988). This mechanism, called the galactic magnetic tornado, is a similar mechanism to that proposed by Uchida and Shibata (1986) for the formation of the galactic center lobe.

\subsection{Explosion Hypothesis}

The vertical structures may alternatively be produced by a sudden energy release at the galactic center such as an explosion or a star burst. In fact molecular and hydrogen line observations of the disk gases exhibit various expanding ring fea- 

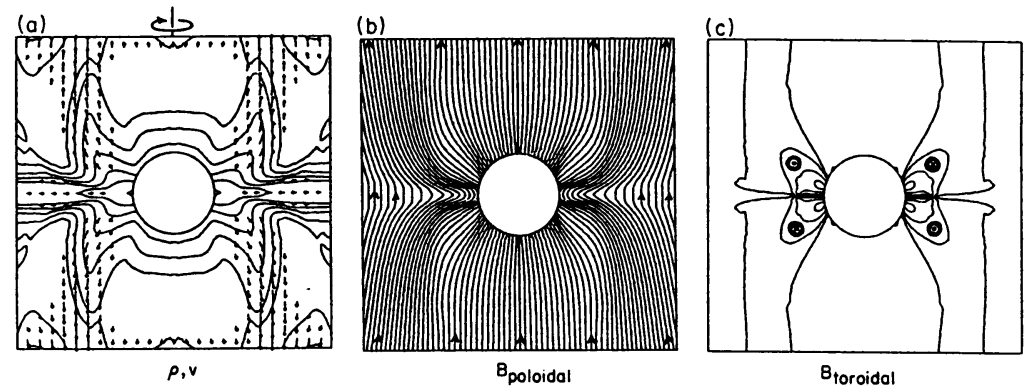

Fig. 7: MHD acceleration of a cylindrical lobe by a twisted poloidal magnetic field by the accretion of disk gas (Shibata and Uchida 1987).
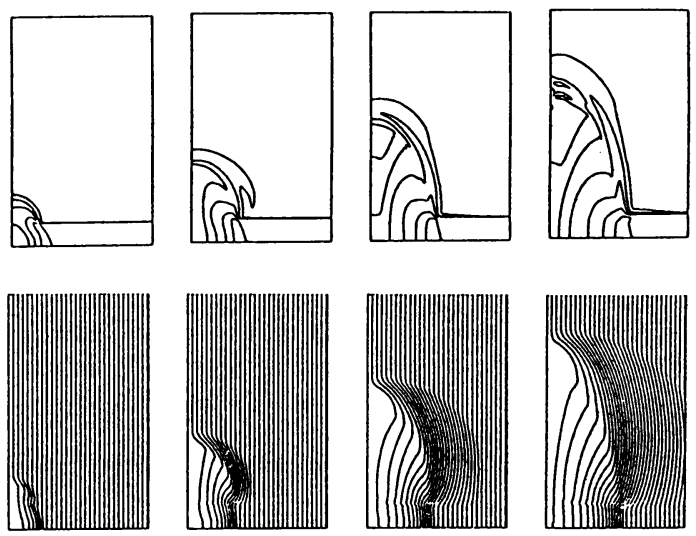

0.07

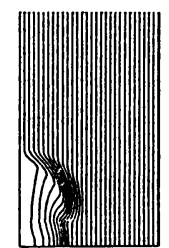

0.17

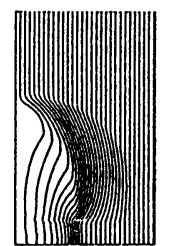

0.34

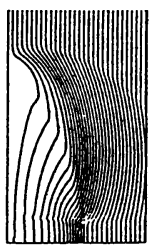

0.50

Fig. 8: Explosion at the center of a gas disk produces a cylindrical lobe structure (Umemura et al 1988).
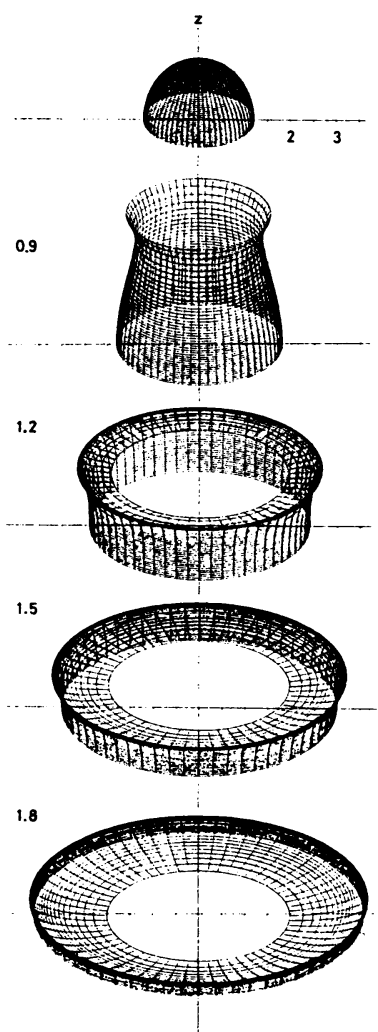

Fig. 9: An MHD wave front produced near the center focuses on a ring of radius $r \sim 2 h$ with $h$ being the scale height of the disk. In case of $h \sim 100 \mathrm{pc}$, we have the radius of the expanding ring as $r \sim 200 \mathrm{pc}$. 
tures. We here discuss the possibility that an explosion at the center will produce the vertical structures and examine if the expanding rings in the disk plane are associated with vertical strucures.

An explosion and an energy release in a short time like a star burst at the center of a rotating disk can be theoretically traced by hydrodynamic and MHD codes. A shock wave which is initially spherical expands more rapidly in the direction perpendicular to the disk plane because of the smaller gas pressure and mass in this direction compared to those in the disk plane. Then the shock front attains an $\Omega$ shape elongated perpendicular to the disk, mimicking the GCL at some stage (Sofue 1977, 1984; Tomisaka and Ikeuchi 1987). If there exists poloidal field the collimation along the rotation axis becomes more pronounced (Umemura et al 1988).

\section{EXPANDING RINGS}

Besides the vertical structures, several expanding ring features of various scales are found in the galactic plane, which are mainly observed in the molecular and hydrogen line emissinos (Oort 1977; Kaifu et al. 1972; Scoville 1972; Tsuboi 1988).

\subsection{Focusing waves}

Further evolution of the shock front following the stage of GCL has been modeled by an MHD wave approximation (Sofue 1977). The wave front initially spherical is elongated to attain a cylindrical shape. Then the waves are refracted to bend toward the galactic plane and focuses on a ring (Fig. 9). The focal ring appears at a radius $r \sim 2 h$, if the variation of the gas density is gaussian with the height from the plane. Here $h$ is the scale height of the disk gas. For $h \sim 100$ pc we have $r \sim 200$ pc. In case that the wave dissipation is negligible and the amplitude is small, approximately $80 \%$ of the energy released at the center explosion focuses on the ring. Moreover the wave may not necessarily disturb the disk gas inbetween the center and the ring during the propagation, and produces an expanding ring by the high-efficiency focusing.

The explosion energy, being transformed by the wave, is then used to accelerate the gas in the focal ring to produce an expanding motion of the ring gas. We note that the ring radius is detemined by the disk thickness, $h$, so that the ring appears always at the same radius, independent of the explosion energy. If the GCL is due to an explosion, it is possible to suppose that the GCL will in future focus on a ring at the same distance, $\sim 200 \mathrm{pc}$, from the center.

The molecular expanding ring of 200 pc radius (Kaifu et al 1972; Scoville 1972: Bally et al 1987) might be explained by this expanding focal ring model. The ring has a radius of $200 \mathrm{pc}$ and is expanding at a velocity of $50 \mathrm{~km} \mathrm{~s}^{-1}$. The ring is situated almost within the galactic plane with little inclination. The kinetic energy of the expansion motion is of the order of $10^{54} \mathrm{ergs}$. This energy is comparable to that involved in the galactic center lobe. The coincidence between the energies in the 200-pc ring and the GCL is consistent if the GCL will focus on the ring in the future, and the 200-pc ring is the consequence of the shock focusing of a past GCL some $10^{6} \mathrm{y}$ ago. The other expanding features as the $30 \mathrm{pc}$-ring (Tsuboi 1988) or the 3-kpc ring (Oort 1977) might also be understood as the result of a similar shock propagation from the center.

\subsection{Cylindrical structure of the ring}

The tangential points to the $200-\mathrm{pc}$ ring on the $l-v$ diagram appear at $l=1^{\circ} .6$ and at $l=-1^{\circ} .2$. The cross section of the ring may be observed if we look at the 


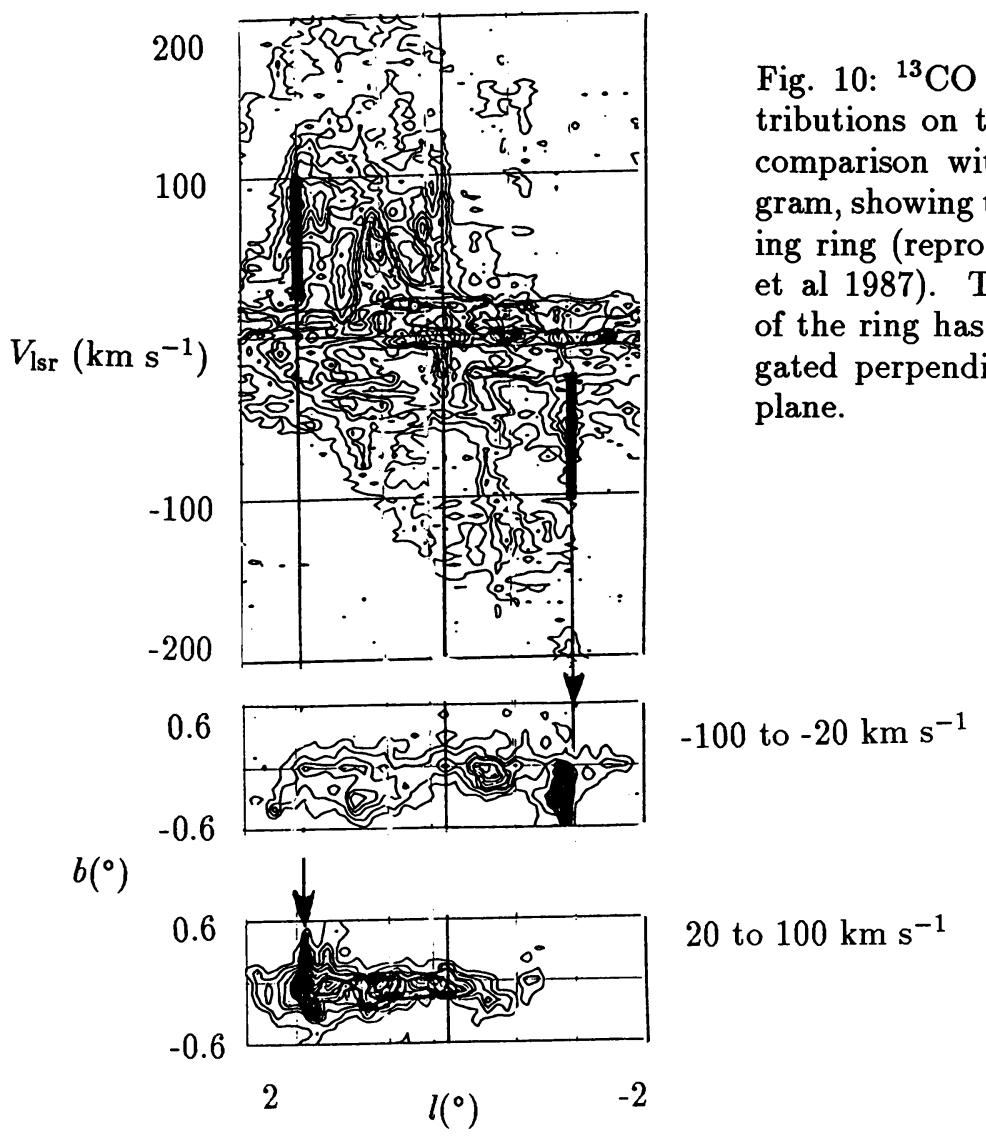

ring toward these directions at the tangential velocities, $v=50 \sim 100 \mathrm{~km} \mathrm{~s}^{-1}$ and $-50 \sim-100 \mathrm{~km} \mathrm{~s}^{-1}$, respectively. Fig. 10 shows the distributions of the $\mathrm{CO}$ gas at these velocities. The cross sections of the ring are seen as the clumps at the above longitudes (as hatched).

The CO clumps are elongated in the direction perpendicular to the disk plane. The ridge extends up to $b \simeq \pm 0^{\circ} .5 \sim \pm 70 \mathrm{pc}$ for the eastern cross section, while it reaches to $b \simeq-0^{\circ} .6$ or lower toward the west. It must be emphasized that the vertical extension is more than the disk thickness which is typically $\pm 0^{\circ} .1-0^{\circ} .2 \sim$ $15-30 \mathrm{pc}$. The 200-pc expanding ring therefore has a cylindrical shape with the vertical extensioin of $\sim 100$ pc. Such a shape is consistent with that expected from a shock propagation models (Tomisaka and Ikeuchi 1987), MHD shock model (Umemura et al 1988) or by the MHD wave model (Sofue 1977). Tsuboi (1988) showed also that the 30-pc ring has a cylindrical structure.

A cylindrical molecular ring has been also found in the starburst galaxy M82 (Nakai et al 1987). The "200-pc ring" in M82 is thought to be the consequence of an 

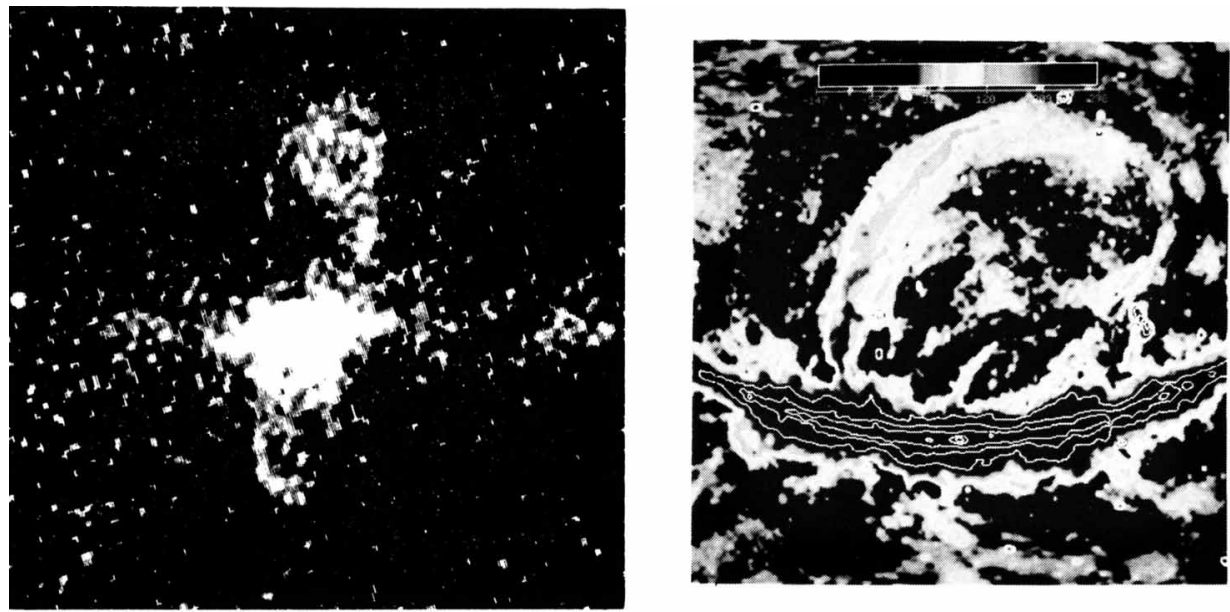

Fig. 11: Radio lobes of a few-kpc scale in the edge-on galaxy NGC 3079 (left panel: Duric et al 1983), and a huge radio lobe in our Galaxy (North Polar Spur) on a $408 \mathrm{MHz}$ background filtered map in curved coordinates so that the NPS is not deformed as in Fig.2.

energy release, while far more intense than that in our Galaxy center, by successive explosions of supernovae in the central region of the dense molecular disk, which is followed by a vertical wind of high-temperature gas as well as the formation of an expanding ring and a cylinder. Existence of vertical magnetic field is not known, while the intense nonthermal radio emission suggests field strength as high as some $10 \mu \mathrm{G}$.

\section{CONCLUDING REMARKS}

Radio continuum observations of the central region of the Galaxy have shown a variety of enigmatic structures elongated in the direction perpendicular to the disk plane. These vertical structures are often associated with magnetic field running parallel to their extensions. The structures may be the manifestation of energy release from the nuclear disk or from the nucleus itself somehow collimated by the vertical magnetic fields.

Two possible mechanisms have been considered: (a) an MHD mechanism in which a twisted poloidal field accelerates the gas perpendicular to the disk. This mechanism can explain a large-scale ejection such as the 4-kpc jet and GCL. Thin filaments like the threads might be some fluctuation of the poloidal field. (b) Alternatively, fronts of shocks and/or MHD waves originating from the nuclear region may cause the vertical extensions. In this mechanism the GCL may be understood as the result of an explosion (or successive supernova explosions). In reality, however, the observed features may be produced by a mixture of the above processes. 
While the vertical features are found near the rotation axis of the galaxy at $r<100 \mathrm{pc}$, the 200-pc expanding ring observed in the molecular lines is found apart from the center at $r \sim 200$ pc. The cross section of the ring also extends in the direction perpendicular to the disk. Such a cylinder, although short, may be due to focusing of MHD waves from the center, being refracted by the disk gas.

Spiral galaxies with active galactic nuclei often exhibit large-scale ejection out of the galactic plane. The edge-on galaxy NGC 3079 posesses symmetrical radio lobes which emanate from the nuclear disk and extend high $(\sim 3-4 \mathrm{kpc})$ above the disk (Fig. 11: Duric et al 1983). The polarization indicates its magnetic characteristics. Similar lobes and jets of $\sim 1-2 \mathrm{kpc}$ emerging perpendicular to disk planes have been found in several edge-on galaxies (Hummel et al 1983).

The vertical structures found in our Galaxy center like the GCL and the Galactic center jet may be similar structures to those found in external galaxies. Besides these we note that the North Polar Spur (NPS; Fig. 2, 11) composes an omega shaped lobe on the sky and is similar to the large lobes in NGC 3079 insofar as its apparent shape is concerned. The NPS was studied on the assumption of a local supernova remnant. However, another possibility that the NPS is structure associated with galactic center activity has not been thoroughly studied: it seems worthwhile to examine such a possibility based on more recent, advanced data and modern analysis.

\section{References}

Altenhoff,W.J., Downes,D., Pauls.,T., Schraml,J. 1979, A. Ap. Suppl., 35, 23

Bally,J., Stark,A.A., Wilson,R.W., Henkel,C. 1987, Ap.J. Suppl., 65, 13

Becklin,E.E. in this issue

Berkhuijsen,E.M., Beck,R., Gräve,R. 1987, in Interstellar Magnetic Fields, ed. R.Beck and R.Gräve (Springer Verlag, Berlin), p.38

Brown,R.L, Liszt,H.S. 1984, Ann. Rev. A. Ap., 22, 223

Downes,D., Goss,W.M., Schwarz,U.J., Wouterloot,J.G.A. 1978, A. Ap. Suppl., 35, 1

Dulk,G.A., Slee,O.B. 1974, Nature, 248, 33

Duric,N., Seaquist,E.R., Crane,P.C., Bignell,R.C., Davis,L.E., 1983, Ap.J. Lett., 273, L11

Ekers,R.D. in this issue

Fujimoto,M., Sawa,T. 1987, Pub. Astr. Soc. Japan, 39, 375

Fujimoto,M., Tosa,M. 1980, Pub. Astr. Soc. Japan, 32, 567

Fürst,E., Sofue,Y, Reich,W. 1987, A. Ap., 191, 303

Genzel,R. in this issue

Genzel,R., Townes,C,H 1987, Ann. Rev. A. Ap., 25, 377

Goss, M. in this issue

Güsten, $R$. in this issue

Güsten,R. 1987, in The Galactic Center, ed. D.Backer (American Inst. Phys. New York), p.19

Güsten,R., Genzel,E., Wright,M.C.H., Jaffe,D.T., Stutzki,J., Harris,A. 1987, Ap.J., preprint

Haslam,C.G.T., Salter,C.J., Stoffel,H., Wilson,W.E., 1982, A. Ap. Suppl. 47, 1

Haslam,C.G.T., Klein,U., Salter,C.J., Stoffel,H., Wilson,W.E., Cleary,M.N., Cooke, D.J., Thomasson,P. 1981, A. Ap., 100, 209 
Handa,T., Sofue,Y., Nakai,N. Inoue,M., Hirabayashi,H. 1987, Pub. Astr.Soc. Japan, 39, 709

Heyvaerts, $J$. in this issue

Heyvaerts,J., Norman,C., Pudritz,R.E., 1987, Ap.J, preprint

Hummel,E., Kotanyi,G.G., van Gorkom,J.H. 1983, Ap.J. Lett., 267, L5

Inoue,M., Fomalont,E., Tsuboi,M., Yusef-Zadeh,F., Morris,M., Tabara,H., Kato,T. in this issue

Inoue,M., Takahashi,T., Tabara,H., Kato,T., Tsuboi,M. 1984, Pub. Astr. Soc. Japan, 36, 633

Kaifu,N., Hayashi,M., Inatani,J., Gatley,I. 1978, in The Galactic Center, ed. D.Backer (American Inst. Phys. New York), p.106

Kaifu,N., Kato,T., Iguchi,T. 1972, Nature, 238, 105

Kapitzky,J.E., Dent,W.A. 1974, Ap.J., 188, 27

Kundt,W. 1987, Ap. Sp. Sci, 129, 195

LaRosa,T.N., Kassim,N.E. 1985, Ap.J. Lett., 299, L13

Lacy,J.H. in this issue

Liszt,H.S. 1985, Ap.J. Letter, 293, L65

Liszt,H.S. 1986, private communication

Little,A.G. 1974, in Galactic Radio Astronomy, IAU Symp. No.60, ed. F.J.Kerr and S.C.Simonson III (D.Reidel, Dordrecht), p.349

Lo,K.Y. in this issue

Lo,K.Y. 1986a, Science, 233, 1394

Lo,K.Y. 1986b, Pub. Astr. Soc. Pacific, 98, 179

Lo,K.Y. 1987, in The Galactic Center, ed. D.Backer (American Inst. Phys. New York), p.30

Mezger,P.G., Pauls,T. 1979, in The Large-scale characteristics of the Galaxy, IAU Symp. No.84, ed. W.B.Burton (D.Reidel, Drodrecht), p.357

Mills,b.Y., Drinkwater,M.J. 1984, Austr.J.Phys., 5, 43

Morris,M. and Yusef-Zadeh,F. 1987, in The Galactic Center, ed. D.Backer (American Inst. Phys. New York), p.127

Morris, M. in this issue

Morris,M., Yusef-Zadeh,F. 1985, A.J., 90, 2511

Nakai,N., Hayashi,M., Handa,T., Sofue,Y., Hasegawa,T., Sasaki,M., 1987, Pub. Astr. Soc. Japan, 39, 685

Okumura et al. in this issue

Oort,J.H. 1977, Ann. Rev. A. Ap, 15, 295

Oort,J.H. 1986, in The Milky Way Galaxy, IAU No.105, eds. H.van Woerden et al (D.Reidel Pub., Dordrecht), p.349

Parker,E.N. 1972, Ap.J., 163, 255

Pauls,T., Downes,D., Mezger,P.G., Churchwell,W. 1976, A. Ap., 46, 407

Reich,W. in this issue

Reich,W., Fürst,E., Steffen,P., Reif,K., Haslam,C.G.T. 1984, A. Ap. Suppl., 58, 197

Reich,W., Sofue,Y., Fürst,E. 1987a, Pub. Astr. Soc. Japan, 39, 573

Reich,W., Sofue,Y., Wielebinski,R., Seiradakis,J.H. 1987b, A.Ap.Suppl. 58, 197

Ruzmaikin,A.A., Sokolov,D.D., Shukurov,A.M. 1985, A. Ap., 148, 335

Rieke,G.H. in this issue

Sawa,T., Fujimoto,M. 1986, Pub. Astr. Soc. Japan, 38, 133

Scoville,N.Z. 1972, Ap.J.Lett., 175, L127 
Seiradakis,J.H., Lasenby,A.N., Yusef-Zadeh,F., Wielebinski,R., Klein,U. 1985, Nature, 317, 697

Serabyn,E., Güsten,R. 1986, A. Ap., 161, 334

Serabyn,E., Güsten,R. 1987, Preprint

Serabyn,E., Lacy,J.H. 1985, A p.J., 293, 445

Shibata, $K$. in this issue

Shibata,K., Uchida,Y. 1987, Pub. Astr. Soc. Japan, 39, 559

Sofue,Y. 1977, A. Ap., 60, 327

Sofue,Y. 1984, Pub. Astr. Soc. Japan, 36, 539

Sofue,Y. 1985, Pub. Astr. Soc. Japan, 37, 697

Sofue,Y. 1988, Pub. Astr. Soc. Japan, in press

Sofue,Y. 1988, Com.on Astr., in press

Sofue,Y., Fujimoto,M. 1987, Ap.J Lett., 319, L73

Sofue,Y., Fujimoto,M. 1988, Pub. Astr. Soc. Japan, in press

Sofue,Y., Fujimoto,M., Wielebinski,R. 1986, Ann. Rev. A. Ap, 24, 459

Sofue,Y., Handa,T. 1984, Nature, 310, 568

Sofue,Y., Inoue,M., Handa,T., Tsuboi,M., Hirabayashi,H., Morimoto,M., Akabane,K. 1986, Pub. Astr. Soc. Japan, 38, 483

Sofue,Y., Reich,W., Inoue,M., Seiradakis,J.H. 1987, Pub. Astr. Soc. Japan, 39, 359

Sofue,Y., Reich,W., Reich.P., 1988, Preprint

Tomisaka,K., Ikeuchi,S. 1987, Ap.J., 330, 695

Tsuboi,M. in this issue

Tsuboi,M., Inoue,M., Handa,T., Tabara,H., Kato,T., Sofue,Y., Kaifu,N. 1986, A. J., 92,818

Uchida,Y., Shibata,K. 1986, Pub. Astr. Soc. Japan, 38,

Uchida,Y., Shibata,K., Sofue,Y. 1985, Nature, 317,699

Umemura,S., Iki,K., Shibata,K., Sofue,Y. 1988, Pub. Astr. Soc. Japan, 40, 25

Watson,M.G., Willingale,R., Grindlay,J.E., Hertz,P. 1981, Ap.J. 250, 142

Weiler,K.W. 1983, in Supernova remnants and their X-ray Emission, IAU No. 101, eds. J.Danziger and P.Gorenstein (D.Reidel Pub., Dordrecht), p.299

Yusef-Zadeh,F. 1986, Ph.D. Thesis, Columbia University, New York

Yusef-Zadeh,F. in this issue

Yusef-Zadeh,F., Morris,M. 1988, Ap.J., preprint

Yusef-Zadeh,F., Morris,M., Chance,D. 1984, Nature, 310, 557

Yusef-Zadeh,F., Morris,M., Slee,O.B., Nelson,G.J. 1986, Ap.J., 310, 689

Yusef-Zadeh,F., Morris,M., Slee,O.B., Nelson,G.J. 1986, Ap.J.Lett., 300, L47 